\title{
LigGrep: a tool for filtering docked poses to improve virtual-screening hit rates
}

\author{
Emily J. Ha' ${ }^{1}$ Cara T. Lwin ${ }^{2}$ and Jacob D. Durrant ${ }^{2^{*}}$ (1)
}

\begin{abstract}
Structure-based virtual screening (VS) uses computer docking to prioritize candidate small-molecule ligands for subsequent experimental testing. Docking programs evaluate molecular binding in part by predicting the geometry with which a given compound might bind a target receptor (e.g., the docked "pose" relative to a protein target). Candidate ligands predicted to participate in the same intermolecular interactions typical of known ligands (or ligands that bind related proteins) are arguably more likely to be true binders. Some docking programs allow users to apply constraints during the docking process with the goal of prioritizing these critical interactions. But these programs often have restrictive and/or expensive licenses, and many popular open-source docking programs (e.g., AutoDock Vina) lack this important functionality. We present LigGrep, a free, open-source program that addresses this limitation. As input, LigGrep accepts a protein receptor file, a directory containing many docked-compound files, and a list of user-specified filters describing critical receptor/ligand interactions. LigGrep evaluates each docked pose and outputs the names of the compounds with poses that pass all filters. To demonstrate utility, we show that LigGrep can improve the hit rates of test VS targeting $H$. sapiens poly(ADPribose) polymerase 1 (HsPARP1), H. sapiens peptidyl-prolyl cis-trans isomerase NIMA-interacting 1 (HsPin1 p), and S. cerevisiae hexokinase-2 (ScHxk2p). We hope that LigGrep will be a useful tool for the computational biology community. A copy is available free of charge at http://durrantlab.com/liggrep/.
\end{abstract}

Keywords: Virtual screening, Computer-aided drug discovery, Computational biology, Filters

\section{Introduction}

Traditional high-throughput screening (HTS) is a powerful experimental technique for identifying molecules that can be further developed into novel pharmaceutics. It involves screening a large number of compounds against selected disease targets (e.g., disease-relevant proteins) to find compounds (i.e., "hits") that elicit measurable biological or biophysical responses. HTS does not require any prior knowledge of the drug-target structure. But the associated hit rates are often low, and it is costly to experimentally test so many molecules. HTS is thus limited primarily to research groups working in the pharmaceutical industry [1].

*Correspondence: durrantj@pitt.edu

${ }^{2}$ Department of Biological Sciences, University of Pittsburgh, Pittsburgh, PA 15260, United States

Full list of author information is available at the end of the article
Computer-aided drug discovery (CADD) aims to address some of these challenges. Among CADD techniques, structure-based virtual screening (VS) is particularly popular. VS draws on a library of virtual small molecules and a model of the drug target. A docking program first predicts how each molecule physically interacts with the target protein, often by binding to a pocket on the target surface [2]. The geometry of the bound molecule relative to the target structure is called the docked pose. Based on this pose, the docking program then estimates the strength of binding (the docking score) using a pre-determined scoring function [3]. The set of compounds with reasonable docked poses and good docking scores is often enriched with true binders. These candidate ligands are then prioritized for subsequent experimental testing [1, 4]. This targeted-search approach

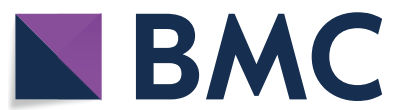

(c) The Author(s) 2020. This article is licensed under a Creative Commons Attribution 4.0 International License, which permits use, sharing, adaptation, distribution and reproduction in any medium or format, as long as you give appropriate credit to the original author(s) and the source, provide a link to the Creative Commons licence, and indicate if changes were made. The images or other third party material in this article are included in the article's Creative Commons licence, unless indicated otherwise in a credit line to the material. If material is not included in the article's Creative Commons licence and your intended use is not permitted by statutory regulation or exceeds the permitted use, you will need to obtain permission directly from the copyright holder. To view a copy of this licence, visit http://creativeco mmons.org/licenses/by/4.0/. The Creative Commons Public Domain Dedication waiver (http://creativecommons.org/publicdomain/ zero/1.0/) applies to the data made available in this article, unless otherwise stated in a credit line to the data. 
reduces cost, preparation time, and workload relative to HTS.

Assessing candidate ligands by docking score is straightforward, but assessing predicted poses is often more subjective and time consuming. One objective pose-assessment approach is to search for new molecules that are predicted to participate in the same critical interactions with the drug target that are typical of known ligands. For example, all clinically approved inhibitors of influenza neuraminidase interact with certain key arginine residues [5], so docked compounds that are not predicted to participate in those interactions might be discarded regardless of score. Unfortunately, in the case of large-scale VS there may be many thousands (or even millions) of docked poses, complicating comprehensive manual inspection.

To automate pose assessment, some commercial docking programs (e.g., Schrödinger's Glide and OpenEye's FRED) automatically reject poses that do not satisfy userspecified filters. These programs are powerful, but they have restrictive and/or expensive licenses that make them inaccessible to many groups. Even OpenEye's free academic license imposes substantial commercialization and intellectual-property restrictions. In contrast, many popular open-source docking programs (e.g., AutoDock Vina [6]) have far more permissive licenses, but they often lack a pose-filtering step.

To address this limitation, we have created LigGrep, a free, open-source program that filters predicted ligand poses that were previously generated during a VS campaign. LigGrep analyzes the pose of each docked compound and discards those poses that do not participate in user-specified interactions. Alternatively, it can also discard poses that involve unfavorable interactions (e.g., interactions with residues known to be involved in resistance mechanisms). LigGrep will help improve VS hit rates by allowing the community to focus on high-scoring compounds that also satisfy carefully chosen binding-pose criteria. A copy is available at http://durrantlab .com/liggrep/, released under the terms of the Apache License, Version 2.0.

\section{Implementation \\ The LigGrep algorithm}

LigGrep accepts as input (1) a PDBQT or PDB file of the drug-target receptor used for docking, (2) a directory of PDBQT, PDB, or SDF files containing the docked poses of candidate ligands, and (3) a JSON-formatted file describing user-specified filters. After evaluating each docked pose, it outputs the names of any compounds with poses that satisfy all user-defined filters. The following sections describe each of these steps in detail.

\section{Input receptor molecule}

LigGrep's first command-line argument is the path to the $\mathrm{PDB} / \mathrm{PDBQT}$-formatted receptor file used for docking. LigGrep uses the Scoria Python library [7] to load the receptor file. Because Scoria is a pure-Python library with a permissive license, we package a copy together with LigGrep itself for convenience.

\section{Input ligand molecules}

LigGrep's second command-line argument is the path to a directory containing the docked-compound files. To accommodate a broad range of docking programs, we designed LigGrep to accept docked poses in three popular file formats. These include the PDBQT format to accommodate docking programs such as AutoDock Vina [6], the PDB format to accommodate big-data studies of crystallographic poses, and the SDF format to accommodate programs such as Schrödinger's Glide. Researchers who wish to examine predicted or experimentally determined poses saved in other formats can convert to the SDF format using open-source programs such as Open Babel [8].

In many cases, users will wish to filter poses by considering only a single ligand atom (e.g., "which poses place an oxygen atom in a particular region of the binding pocket?"). In other cases, users may wish to filter poses using more complex ligand substructures (e.g., "which poses place a hydroxyl group in a particular region?"). In still other cases, substructures with specific atomic-bond specifications may be critical (e.g., "which poses place a phenyl group-but not a cyclohexyl group-in a particular region?"). Users can run LigGrep in three modes (NONE, OPENBABEL, and SMILES) according to their needs.

NONE mode. In NONE mode (--mode NONE), LigGrep does not assign bond orders beyond those described in the docked-compound files themselves. In the case of PDB/PDBQT files, which do not include any information about bond orders, LigGrep assumes that all atoms are $\mathrm{sp}^{3}$ hybridized and that all appropriately juxtaposed atoms are connected by single bonds. In the case of SDF files, which do include detailed information about atomic bonds, LigGrep instead assigns bond orders based on the information present in the SDF files themselves. NONE mode is thus ideal when (1) filtering by a single atom, (2) filtering by a substructure whose atoms are connected only by single bonds, or (3) applying filters of any type to SDF-formatted docked poses.

SMILES mode. In SMILES mode (--mode SMILES), LigGrep assigns bond orders to PDB/PDBQT-formatted docked-compound files by additionally considering user-specified files in the SMILES format. The simplified molecular-input line-entry system (SMILES) format is a 
widely used, compact format that encodes a molecule's connectivity and chirality as a simple, one-line string of letters and other symbols. To run LigGrep in SMILES mode, the user should save a separate SMILES file for each PDB/PDBQT-formatted docked compound, in the same compound directory. Each SMILES file should have the same filename as the corresponding PDB/PDBQT file, plus the "smi" extension. SMILES mode is ideal when (1) SMILES strings are available or can be easily generated, (2) filters involve substructures with higherorder (e.g., double, triple, aromatic) bonds, and (3) the docked files are PDB/PDBQT formatted. SMILES mode is not appropriate for SDF-formatted files because these already include bond-order information.

OPENBABEL mode. In OPENBABEL mode (--mode OPENBABEL), LigGrep uses the Open Babel (obabel) executable [8] to try to assign atom hybridization and bond orders to docked-compound PDB/PDBQT files. Users must specify the path to the Open Babel executable using LigGrep's --babel_exec /PATH/TO/OBABEL parameter. Internally, Open Babel converts PDB/PDBQT files to the SDF format, which includes bond-order information that the Python library RDKit [9] can then process. Unfortunately, PDBQT files do not include nonpolar hydrogen atoms, complicating this process. LigGrep runs Open Babel first using the $-h$ option to add all missing hydrogen atoms. If that hydrogen-atom assignment does not match the atoms present in the input PDB/PDBQT file, LigGrep reruns Open Babel using the $-p$ option, which adds hydrogen atoms as appropriate for neutral $\mathrm{pH}$ (7.4). If this second attempt fails, LigGrep issues a warning and moves on to the next docked-compound file.

We provide OPENBABEL mode for user convenience. It allows users to process $\mathrm{PDB} / \mathrm{PDBQT}$-formatted poses using higher-order substructure filters, even when SMILES strings are not available. But we recommend using OPENBABEL mode cautiously. Given the ambiguities associated with assigning hybridization and bond orders based on atomic coordinates alone, LigGrep in OPENBABEL mode may misclassify some compounds. Furthermore, OPENBABEL mode is not appropriate when filtering SDF-formatted poses because SDF files already include bond-order information.

\section{Input-filters file}

LigGrep's third command-line argument is the path to a JSON file containing a list of filters that the input compounds must satisfy (Fig. 1). LigGrep filters have four user-defined components: (1) a ligand-substructure specification describing one or more bonded atoms,
(2) a point in 3D space (the query point), (3) a distance cutoff, and (4) an optional "exclude" flag.

Identifying ligand substructures. To determine whether a given docked pose satisfies the user-specified filters list, LigGrep first uses the RDKit Python library [9] to check whether the molecule contains the necessary substructures (i.e., the substructures associated with all filters that do not have "exclude" flags, Fig. 1a). LigGrep rejects all molecules that do not contain each of the necessary substructures. Users specify substructures via SMILES arbitrary target specification (SMARTS) notation (Fig. 1a and d), which is syntactically similar to SMILES. When using LigGrep in NONE mode to filter PDB/PDBQT-formatted docked molecules, substructures must include only $\mathrm{sp}^{3}$-hybridized atoms connected by single bonds (as in Fig. 1a). Otherwise, the SMARTS strings can include more complex substructure descriptions (e.g., aromatic rings, as in Fig. 1d).

Identifying the 3D query point. For each specified filter, LigGrep identifies the appropriate 3D query point by examining the corresponding JSON data. If the filter JSON contains the key "coordinate", LigGrep constructs the $3 \mathrm{D}$ query point directly from the corresponding value, a list containing $x, y$, and $z$ coordinates (Fig. 1e). If the filter JSON contains the key "receptorAtom", LigGrep constructs the $3 \mathrm{D}$ query point by searching the input receptor file for an atom that matches the provided chain, residue id (resid), and atom name (atomname) (Fig. 1b). The 3D query point is then set to the coordinate of that atom.

Accepting or rejecting docked compounds based on atomic distances. Once LigGrep has identified the relevant small-molecule substructures and 3D query points, it determines whether a given docked pose passes 
or fails each filter. If it passes all filters, the name of the compound file is saved to an output text file. If it fails any of the filters, the compound file name is not saved. The filters are thus combined using a Boolean AND (conjunction) operator, though advanced users familiar with SMARTS notation can also embed additional logical operators within their substructure specifications for more complex, nested logic.

To apply each filter, LigGrep first calculates the minimum distance between any small-molecule-substructure atom and the corresponding 3D query point. It then compares this calculated distance to the cutoff distance associated with each filter (Fig. 1c). By default, a given docked pose passes a filter if it positions the substructure near the query point. If the user includes an optional "exclude" flag (Fig. 1f), a pose passes only if it does not position the substructure near the query point. The specific criteria used to assess each filter are given in Table 1.

\section{Dependencies and compatibility}

We have tested LigGrep on macOS, Ubuntu Linux, and Windows 10 (Table 2). LigGrep requires the third-party Python libraries RDKit [9], NumPy [10], and SciPy [11], which must be installed separately. It comes pre-packaged with the Scoria library [7] for convenience. When running LigGrep in the preferred NONE or SMILES mode, the Open Babel executable is not required. Users who wish to use OPENBABEL mode must install Open Babel separately.

Table 1 LigGrep criteria for assessing user-defined filters

\begin{tabular}{llll}
\hline Has substructure & $\begin{array}{l}\text { Exclude flag } \\
\text { set }\end{array}$ & $\begin{array}{l}\text { Any substructure atom } \\
\text { within cutoff distance }\end{array}$ & Result \\
\hline No & No & N/a & Fail \\
No & Yes & N/a & Pass \\
Yes & No & No & Fail \\
Yes & No & Yes & Pass \\
Yes & Yes & No & Pass \\
Yes & Yes & Yes & Fail \\
\hline
\end{tabular}

\section{Benchmark virtual screens}

\section{Preparing the receptors}

To demonstrate LigGrep use, we performed VS against three proteins: $H$. sapiens poly(ADP-ribose) polymerase 1 (HsPARP1), H. sapiens peptidyl-prolyl cis-trans isomerase NIMA-interacting 1 (HsPin1p), and S. cerevisiae hexokinase-2 ( $S c \mathrm{Hxk} 2 \mathrm{p})$. We downloaded HsPARP1, $H s$ Pin1p, and $S c H x k 2 p$ crystal structures (6BHV [12], 3TDB [13], and 1IG8 [14], respectively) from the RCSB Protein Data Bank $[15,16]$. We selected the 6BHV $H s$ PARP1 structure because its co-crystallized ligand, benzamide adenine nucleotide, was the largest by mass of 46 co-crystallized ligands considered (Additional file 1: Table S1), and some studies suggest that larger bindingpocket conformations are more amenable to VS $[17,18]$. We selected the 3TDB HsPin1p structure because its cocrystallized ligand was the largest by mass of 27 considered (Additional file 1: Table S2). Finally, we selected the 1IG8 $S c H x k 2 p$ structure because it is the only $S c H x k 2 p$ structure with the correct amino-acid sequence. In all cases, we used the PDB2PQR server [19-21] to add hydrogen atoms to these protein structures and to optimize their hydrogen-bond networks (default parameters, $\mathrm{pH}$ 7). We then used Open Babel [8] to convert the resulting protonated $\mathrm{PQR}$ files to the $\mathrm{PDB}$ format. Finally, we used MGLTools 1.5.6 [22] to convert the PDB files to the PDBQT format, which includes atom types and partial atomic charges.

\section{Preparing the small-molecule libraries}

To prepare a library of small molecules for HsPARP1 docking, we downloaded the SMILES strings of 46 known HsPARP1 ligands (Additional file 1: Table S1) and 1515 diverse small molecules (presumed decoys). The known ligands were taken from HsPARP1 crystal structures deposited in the RCSB Protein Data Bank, and the decoys were taken from the NCI Diversity Set VI, a set of freely available compounds provided by the National Cancer Institute (NCI) [23]. We created a similar smallmolecule library of known ligands and NCI decoys for $H s$ Pin1p docking, using 27 co-crystallized HsPin1p ligands (Additional file 1: Table S2). For ScHxk2p

Table 2 We have tested LigGrep on various operating systems, using various versions of Python 3, RDKit, NumPy, SciPy, and Open Babel

\begin{tabular}{|c|c|c|c|c|c|c|}
\hline Operating system & Python & RDKit & NumPy & SciPy & Open babel & Multiprocessing \\
\hline macOS Mojave 10.14.5 & 3.6 .7 & 2019.03 .3 & 1.17 .4 & 1.3 .1 & 2.4 .1 & Y \\
\hline Ubuntu 18.04.4 LTS & 3.6 .5 & 2019.03.1 & 1.16 .3 & 1.3 .0 & 3.1 .0 & Y \\
\hline Ubuntu 18.04.2 LTS & 3.7 .3 & 2020.03 .3 & 1.18 .1 & 1.4 .1 & 2.3 .2 & Y \\
\hline Windows 10 Home 1903 & 3.7 .4 & 2020.03 .2 & 1.16 .5 & 1.3 .1 & 2.3 .1 & $\mathrm{~N}$ \\
\hline
\end{tabular}

Multiprocessing is not supported on Windows. Note that LigGrep is designed to work with Python 3, not Python 2 
docking, we identified 41 glucose analogues known to bind hexokinase and glucokinase proteins from various species (presumed $S c H x k 2 p$ ligands; eight from the RCSB Protein Data Bank, and 33 from the BindingDB database [24, 25]; Additional file 1: Table S3). As $S c H x k 2 p$ decoys, we selected 1652 glucose analogues with molecular weights less than 500 Daltons, taken from the ChemDiv and eMolecules databases (presumed inactives).

We used the open-source program Gypsum-DL [4] to generate 3D small-molecule models from these SMILES strings. To account for alternate ionization, tautomeric, chiral, isomeric, and ring-conformational forms, we instructed Gypsum-DL to generate two molecular variants per input compound (min_ph: 7.4; max_ph: 7.4; pka_precision: 0). We also used Gypsum-DL's --use_durrant_lab_filters flag to remove molecular variants judged improbable. The output small-molecule PDB files were again converted to the PDBQT format using MGLTools 1.5.6 [22].

\section{Docking}

To prepare for docking, we used AutoDockTools [22] and Webina [26] to identify a docking box centered on the ligand-binding pockets of the respective crystal structures. In the case of the 6BHV HsPARP1 structure, we retained all four catalytic domains present in the $6 \mathrm{BHV}$ asymmetric unit for simplicity's sake, but the docking box (23 $\AA$ x $17 \AA$ x $20 \AA$ ) encompassed only chain- $A$ atoms. The $H s$ Pin $1 p$ and $S c H x k 2 p$ docking boxes were $20 \AA$ x 20 $\AA$ x $20 \AA$ and $20 \AA$ x $19 \AA$ x $15 \AA$, respectively. We docked all compounds into their respective receptors using AutoDock Vina [6], with Vina's default parameters.

\section{Defining LigGrep filters}

To construct LigGrep filters suitable for the HsPARP1 VS,PARP1 VS, we reviewed a recently published table of predicted $H s$ PARP1/ligand interactions that were frequently identified in a large-scale de novo CADD campaign [27]. Two of the most frequent interactions were also observed in crystal structures of HsPARP1 bound to the clinically approved inhibitor niraparib (4R6E:A $[28]$ ) and a 4(3H)-quinazolinone derivative (1UK0:A [29]): a hydrogen bond with G863, and a $\pi-\pi$ interaction with Y907. Based on our analysis of these structures, we defined two structural ("receptorAtom") filters to capture the two interactions. To capture the hydrogen bond, we required that a docked-compound nitrogen or oxygen atom (SMARTS: $[\# 7, \# 8]$ ) come within $5.5 \AA$ of the G863 alpha carbon (resid: 863; atomname: CA). To capture the $\pi-\pi$ interaction, we required that a docked-compound carbon-carbon aromatic bond (SMARTS: $c c$ ) come within $5.5 \AA$ of the most distal Y907 carbon atom (resid: 907; atomname: $\mathrm{CZ}$ ).
To construct filters suitable for the $H s$ PARP1 VS, we examined multiple co-crystallized ligands deposited in the RCSB Protein Data Bank. Many of these positioned peptide-backbone-like substructures (e.g., imidazole- and furan-carboxylic-acid moieties) near where endogenous peptides bind [30]. We therefore defined one "coordinate" filter to identify docked poses that positioned [N,O] CCO substructures within $4.0 \AA$ of this position. We used a SMARTS string with only $\mathrm{sp}^{3}$-hybridized atoms (single bonds) to ensure compatibility with LigGrep's NONE mode.

To construct filters suitable for the $H s$ Pin1p VS, we again considered known ligands. Many hexokinase ligands are glucose analogues, so we defined a single "coordinate" filter to identify docked poses that positioned a tetrahydro- $2 \mathrm{H}$-pyran moiety (C1OCCCC1) within $1.0 \AA$ of the 3D position where glucose typically binds.

\section{Measuring virtual screen performance}

To evaluate the performance of our VS in terms of both the docked poses and the associated docking scores, we first selected a single candidate pose for each unique input molecule in our library. Given that Gypsum-DL generated up to two molecular variants for each molecule and that Vina predicted up to 9 poses for each variant, each unique molecule was associated with at most 18 poses. In practice this number was smaller on average because in some cases Gypsum-DL generated only one variant, and LigGrep (when applied) filtered out those poses that failed to meet our user-specified criteria. To assess the VS before applying LigGrep, we considered only each ligand's single, top-scoring pose, without regard for pose geometry. To assess the VS after applying LigGrep, we considered the top-scoring pose from among the poses with geometries that matched our userdefined LigGrep filters.

Pose accuracy. To assess how well Vina predicts the poses of known HsPARP1 ligands, we used UCSF Chimera [31] to align the 46 apo HsPARP1 crystal structures listed in Additional file 1: Table S1 to the HsPARP1 structure used for docking. We then used the obrms program, included in the Open Babel package [8], to calculate the root-mean-square deviation (RMSD) between the topscoring docked pose of each known ligand and the corresponding crystallographic pose (Additional file 1: Table S1). We used this same protocol to assess how well Vina predicts the poses of known $H s$ Pin $1 \mathrm{p}$ and $S c H x k 2 p$ ligands (Additional file 1: Tables S2 and S3), though in the case of $S c H x k 2 p$ only one crystallographic ligand was available.

Scoring (ranking) accuracy. We used several methods to assess how well the three VS ranked known 
ligands above decoy molecules, both before and after applying LigGrep. First, we ordered the compounds of the three VS by their docking scores and calculated the percentile ranks of the known ligands (Additional file 1: Tables S1-S3). Second, we counted the number of positive-control compounds that ranked in the top 10, 20, and 40 compounds for each VS (Table 3). Third, we calculated enrichment factors. Given a VS of $T$ total small molecules including $P_{T}$ positive-control compounds, the enrichment factor, $E F_{n}$, of the $n$ top-ranked compounds is the number of positive-control ligands present, $P_{n}$, divided by the number of positive controls that would be expected if the compounds were randomly ordered, i.e., $E F_{n}=P_{n} /\left(n P_{T} / T\right)$. Finally, though LigGrep is best used to enrich the set of top-ranked compounds, we also assessed the impact of LigGrep filters on the entire set of ranked compounds using receiver operating characteristic (ROC) metrics (see Additional file 1: Figure S1).

\section{Results and discussion}

\section{Benchmark virtual screen: HsPARP1}

To demonstrate utility, we first used LigGrep to enrich a VS targeting the $H s$ PARP1 catalytic pocket. $H s$ PARP1 is a highly conserved, multifunctional enzyme that plays important roles in the DNA-damage response. It posttranscriptionally attaches a negatively charged polymer termed poly(ADP-ribose) (PAR) to various protein targets (including itself). These PAR chains recruit proteins that contribute to DNA repair, to the stabilization of DNA replication forks, and to the modification of chromatin structure [32]. HsPARP1 is over-expressed in various carcinomas, making it a potential therapeutic target. Additionally, multiple preclinical research studies and clinical trials demonstrate that HsPARP1 inhibition can repress tumor growth and metastasis [33].

Although a few HsPARP1 inhibitors have been approved for clinical use (e.g., olaparib, niraparib, and rucaparib) [34], clinical trials have revealed a number of therapeutic limitations. These limitations include (1)

Table 3 The number of known ligands caught in the top 10,20 , and 40 highest-scoring compounds of each VS when docking with Vina only vs. Vina + LigGrep

\begin{tabular}{lllll}
\hline Protein & Screen type & Top 10 & Top 20 & Top 40 \\
\hline HsPARP1 & Vina only & $5 / 46$ & $7 / 46$ & $13 / 46$ \\
HsPARP1 & Vina + LigGrep & $7 / 46$ & $11 / 46$ & $19 / 46$ \\
HsPin1p & Vina only & $2 / 27$ & $3 / 27$ & $5 / 27$ \\
HsPin1p & Vina + LigGrep & $3 / 27$ & $4 / 27$ & $8 / 27$ \\
ScHxk2p & Vina only & $2 / 41$ & $4 / 41$ & $6 / 41$ \\
ScHxk2p & Vina + LigGrep & $4 / 41$ & $6 / 41$ & $7 / 41$ \\
\hline
\end{tabular}

toxicity due to promiscuous binding, given that most HsPARP1 inhibitors resemble NAD+ [35]; (2) activation of viral replication, especially the replication human T-cell lymphotropic virus (HTLV) or Kaposi's sarcomaassociated herpes virus (KSHV) [35]; and (3) acquired resistance that limits long-term use [36]. There is thus an urgent need for novel ligands that can be further developed into clinically useful $H s$ PARP1 inhibitors.

\section{Unfiltered HsPARP1 virtual screen}

We first performed a standard VS on HsPARP1 to establish baseline performance. The screen involved 46 cocrystallized (known) HsPARP1 catalytic inhibitors, as well as 1515 additional molecules that served as decoys (presumed inactives). We evaluated our Vina-based docking protocol both in terms of pose-prediction accuracy and the ability to distinguish between known inhibitors and decoy molecules. To evaluate the accuracy of the predicted poses, we calculated heavy-atom RMSDs between the top-scoring docked and crystallographic poses of the 46 known inhibitors included in our smallmolecule library. The average RMSD value was $2.82 \AA$ ( \pm $2.04 \AA$ stdev), ranging from $0.48 \AA$ (4OPX:A) to $12.14 \AA$ (4HHY:A). Thirty-one of the known HsPARP1 inhibitors had top-scoring docked poses within $3 \AA$ of the crystallographic pose (Additional file 1: Table S1). These RMSD calculations indicate that Vina is reasonably adept at posing the known ligands correctly.

To evaluate how well the Vina-based docking protocol can distinguish between known inhibitors and decoy molecules, we ranked the compounds of our small-molecule library by the docking scores of their top-scoring poses. We found that 45 of 46 known catalytic inhibitors ranked in the top $40 \%$, and 28 ranked in the top $10 \%$. The single highest-scoring compound was in fact a known HsPARP1 catalytic inhibitor ("compound 33" from the 4HHY structure [37]); five known ligands were in the top 10 compounds, seven in the top 20, and 13 in the top 40 (Table 3 and Additional file 1: Table S1).

\section{LigGrep-filtered HsPARP1 virtual screen}

To show how LigGrep can further improve the hit rate of this high-performing VS, we filtered the docked poses of all library compounds to identify those predicted to participate in a hydrogen bond with the HsPARP1 G863 residue and a $\pi-\pi$ interaction with Y907 (Table 3, see "Implementation" for details). LigGrep filtered out 435 of the 1561 unique compounds (ligands + decoys) in the virtual library. We ranked (by docking score) the remaining 1126 unique compounds with poses that matched our filter criteria.

Importantly, LigGrep allowed us to effectively consider all the poses associated with each docked compound, 
not just the top-scoring pose. Given our Gypsum-DL/ Vina protocol (see "Implementation") [4, 6], each unique compound in our library was associated with up to 18 predicted poses. Manually inspecting so many poses is impossible. Our approach in the past has been to consider only the top-scoring pose associated with each Vina run, and then to visually inspect only the poses of the top-ranked docked compounds. LigGrep now allows us to consider all docked poses associated with each candidate ligand, not just the top-scoring pose.

After we used LigGrep to filter out less-likely poses, seven known ligands ranked in the top 10 highestscoring compounds, 11 in the top 20, and 19 in the top 40 (Table 3 and Additional file 1: Table S1). In fact the top four ranked compounds were all known HsPARP1 inhibitors. LigGrep thus improved the hit rate among the top-scoring compounds over that obtained using the unfiltered VS. Similar improvements can be seen in enrichment factors (Fig. 2) and areas under the pROC curve (Additional file 1: Figure S1 and Table S4).

\section{Benchmark virtual screen: $H s P i n 1 p$}

To further demonstrate utility, we also applied LigGrep to a VS targeting $H s$ Pin1p. $H s$ Pin1p binds to proteins with phosphorylated serine/threonine-proline (pSer/ Thr-Pro) motifs and accelerates the cis-trans isomerization of the proline residue. Peptidyl-prolyl isomerization can be seen as a kind of post-translational modification that impacts how many proteins fold, localize, activate, and interact [38-41]. HsPin1p is upregulated in many cancers, likely because many of its targets contribute to cancer pathogenesis. Reduced $H s$ Pin 1 p activity protects against cancer progression [38, 42-44], so much effort has been invested in developing $H s P i n 1 p$ inhibitors [38].

\section{Unfiltered HsPin $1 p$ virtual screen}

As with $H s$ PARP1, we first performed a standard $H s$ Pin1p VS to establish baseline performance. The screen involved 27 co-crystallized (known) HsPin1p inhibitors, as well as 1515 additional molecules that served as decoys (presumed inactives). The average heavy-atom RMSD between the top-scoring docked and crystallographic poses of the 27 known inhibitors was $3.43 \AA$ ( $\pm 2.33 \AA$ stdev), ranging from $0.26 \AA$ (2XP6:A) to 8.63 $\AA$ (3TDB:A). Fourteen of the known $H s$ Pin 1 p inhibitors had top-scoring docked poses within $3 \AA$ of the crystallographic pose (Additional file 1: Table S2).

We again ranked the compounds of our small-molecule library by the docking scores of their top-scoring poses. We found that 23 of 27 known inhibitors ranked in the top $40 \%$, and 12 ranked in the top $10 \%$. Two known ligands were in the top 10 compounds, three in the top
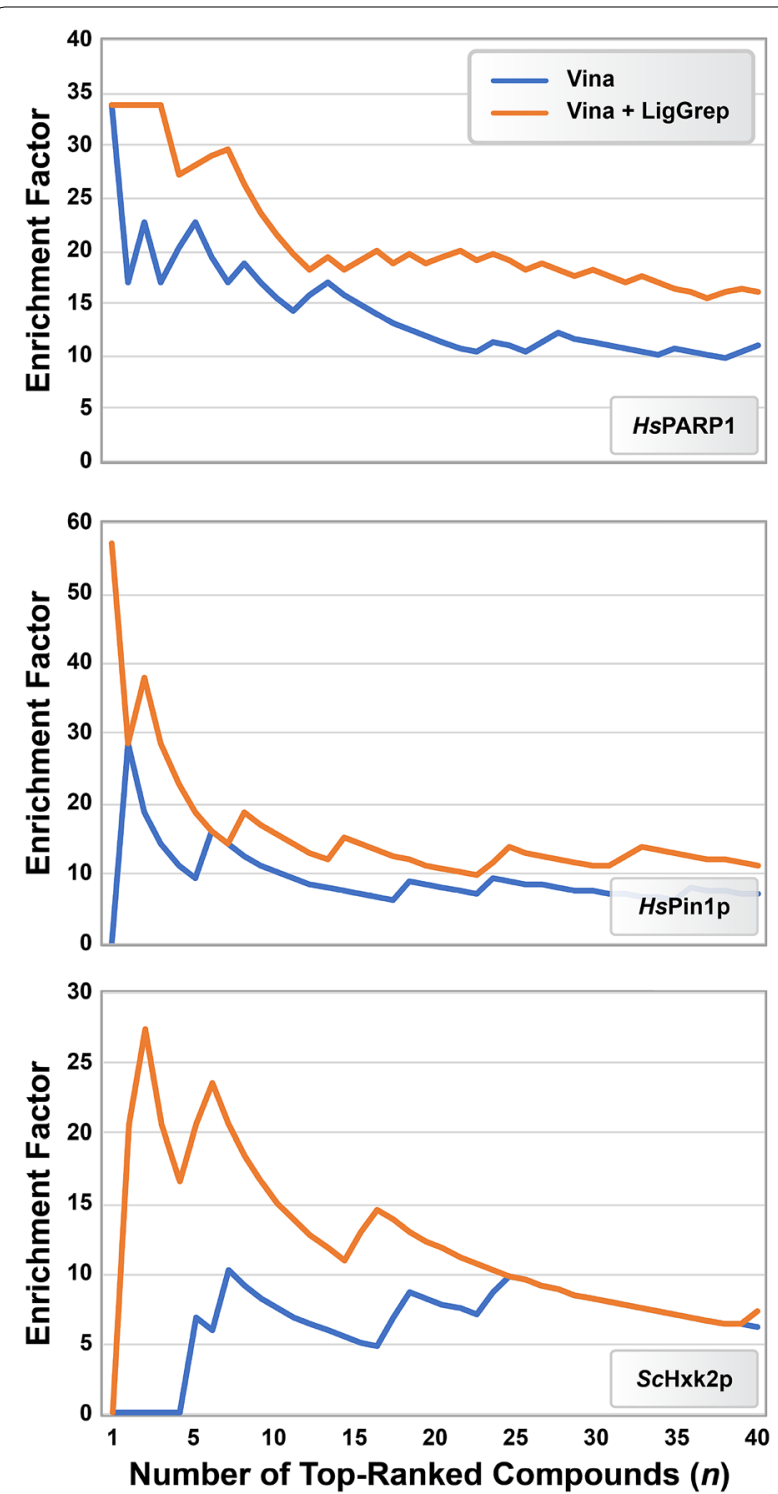

Fig. 2 Enrichment factors associated with our HsPARP1,HsPin $1 p$, and ScHxk2p VS, before (blue) and after (orange) applying LigGrep filters.

To calculate the enrichment factors of the LigGrep-filtered VS, any compound that did not pass the filter(s), whether a positive control or decoy, was moved to the bottom of the ranked list

20, and five in the top 40 (Table 3 and Additional file 1: Table S2).

\section{LigGrep-filtered HsPin $1 p$ virtual screen}

To show how LigGrep can further improve the hit rate of the $H s$ Pin1p VS, we filtered the docked poses of all library compounds to identify those that positioned peptide-backbone-like substructures near where endogenous peptides bind (Table 3, see "Implementation" for details) [30]. Given that this substructure is very specific, 
LigGrep filtered out 1029 of the 1542 unique ligands and decoys. We ranked (by docking score) the remaining 513 unique compounds with poses that matched our filter criteria, including 25 of the known ligands.

After LigGrep filtering, three known ligands ranked in the top 10 highest-scoring compounds, four in the top 20, and eight in the top 40 (Table 3 and Additional file 1: Table S2). LigGrep thus improved the hit rate among the top-scoring compounds. In fact the topranked compound after LigGrep filtering was the known $H_{s}$ Pin1p inhibitor 3-(6-fluoro-1H-benzimidazol-2-yl)-N(naphthalen-2-ylcarbonyl)-D-alanine, with a measured $\mathrm{K}_{\mathrm{i}}$ of $80 \mathrm{nM}$ [45]. The enrichment factors (Fig. 2) and areas under the pROC curve similarly improved (Additional file 1: Figure S1 and Table S4).

\section{Benchmark virtual screen: ScHxk2p}

As a final demonstration, we applied LigGrep to a VS targeting $S c H x k 2 p$. Hexokinases perform the first step in glucose metabolism, transferring an ATP $\gamma$ phosphate to a glucose $\mathrm{C6}$ carbon atom to produce glucose-6-phosphate (Glc-6P) [46]. This activated Glc-6P is critical for downstream catabolic processes such as anaerobic fermentation [47] and aerobic oxidative phosphorylation (OXPHOS) [47], as well as anabolic pathways such as the pentose-phosphate shunt [48-50].

Glucose metabolism is often dysregulated in cancer. Cancer cells tend to use glycolysis and lactic acid fermentation to generate ATP from glucose, even when adequate oxygen is available for the more efficient OXPHOS [49, 51-55]. To maintain ATP levels, cancer cells must increase glycolytic flux [50], often by upregulating hexokinase II (HsHK2p) [48, 49, 56, 57]. Several groups have developed small-molecule $H s \mathrm{HK} 2 \mathrm{p}$ ligands that bind to the catalytic pocket $[48,58-63]$, but acquired resistance [64-71] and unacceptable toxicities [72] require the development of additional hexokinase inhibitors.

\section{Unfiltered ScHxk2p virtual screen}

We first performed a standard $S c H x k 2 p$ VS to establish baseline performance. We designed this VS to determine whether LigGrep can enhance performance even in challenging circumstances. First, rather than limit our set of positive controls ("known ligands") to ScHxk2p ligands, we selected 41 glucose analogues known to bind hexokinases and glucokinases from any of several species. It is therefore likely that some of our "positive controls" are not true $S c \mathrm{Hxk} 2 \mathrm{p}$ ligands, effectively injecting noise into our VS signal. To further exacerbate this challenge, we selected molecules that are chemically similar to the positive controls to serve as presumed inactive decoys (i.e., 1652 glucose analogues present in the ChemDiv and eMolecules databases).

Second, we of necessity had to limit our pre-VS assessment of pose accuracy. Only one of the selected positivecontrol compounds (ortho-toluoylglucosamine [73]) has been co-crystallized with $S c H x k 2 p$. The heavy-atom RMSD between the top-scoring and crystallographic poses of this compound was $1.90 \AA$, suggesting that Vina is well suited to $S c H x k 2 p$ docking, but the available structures permit only this one data point as validation.

We again ranked the compounds of our small-molecule library by the docking scores of their top-scoring poses. We found that 26 of 41 positive-control compounds ranked in the top $40 \%$, and 12 ranked in the top $10 \%$. Two were in the top 10 compounds, four in the top 20, and six in the top 40 (Table 3 and Additional file 1: Table S3).

\section{LigGrep-filtered ScHxk2p virtual screen}

To show how LigGrep can improve the hit rate of the $S c$ Hxk2p VS, we filtered the docked poses of all library compounds to identify those that positioned a glucoselike (tetrahydro- $2 H$-pyran) moiety near the location where glucose, the endogenous substrate, binds (Table 3 , see "Implementation" for details) [74]. LigGrep filtered out 428 of the 1693 unique compounds in the virtual library. We ranked (by docking score) the remaining 1265 unique compounds with poses that matched our filter criteria, including 40 of the positive-control compounds. After LigGrep filtering, the number of positive controls in the top 10 highest-scoring compounds doubled to four. Six positive controls ranked in the top 20, and seven in the top 40 (Table 3 and Additional file 1: Table S3). The enrichment factors (Fig. 2) and areas under the pROC curve also improved (Additional file 1: Figure S1 and Table S4).

\section{LigGrep advantages and disadvantages}

To illustrate the advantages and disadvantages of the LigGrep approach, we now consider in detail several of the docked poses from our HsPARP1 VS.

\section{Examples that illustrate LigGrep advantages}

LigGrep can improve hit rates by (1) eliminating compounds that are less likely to bind the target protein, and (2) allowing researchers to consider all poses (rather than only the top-scoring pose) when searching for potential ligands. The low-nanomolar HsPARP1 inhibitor olaparib [28] illustrates the first advantage (Fig. 3a). The docked and crystallographic poses of olaparib were similar (RMSD: 2.7 Å; Fig. 3a in green and pink, respectively) [75]. Prior to LigGrep filtering, olaparib ranked third in 
our VS $(-12.1 \mathrm{kcal} / \mathrm{mol})$, behind a presumed decoy in second place. But none of the poses associated with the second-place compound passed the filters, so olaparib moved from third to second.

Tricinolone acetophenonide (NSC37641), a high-scoring decoy molecule, illustrates the second advantage of the LigGrep approach (Fig. 3b). The top-scoring pose of this compound (in pink) has a Vina docking score of $-10.4 \mathrm{kcal} / \mathrm{mol}$, but it does not position an oxygen or nitrogen atom close enough to the HsPARP1 G863 residue, as required by one of our LigGrep filters. Had we manually inspected only the top-scoring pose associated with each ligand, we may therefore have discarded this compound, despite its impressive docking score (top $\sim 3 \%$ of all compounds). But LigGrep allowed us to identify a second, slightly lower-scoring pose $(-10.2 \mathrm{kcal} /$ $\mathrm{mol}$ ) that did in fact position a compound hydroxyl group near the G863 residue. Of course, tricinolone acetophenonide is not known to inhibit HsPARP1, and it was in fact included in the screen as a decoy (presumed inactive) compound. But this example nevertheless illustrates how LigGrep allows researchers to identify reasonable albeit lower-scoring docked poses, even when the top-scoring pose is implausible.

\section{Examples that illustrate LigGrep disadvantages}

By way of disadvantages, we note that (1) some incorrect poses nevertheless pass LigGrep filters, and (2) some true ligands may not contain the substructures required to pass, regardless of pose accuracy. "Compound 33," a lownanomolar benzo[de][1,7]naphthyridin-7 $(8 H)$-one inhibitor [37], illustrates the first disadvantage. Though it was the top-scoring compound in our VS $(-13.4 \mathrm{kcal} / \mathrm{mol})$ and passed our LigGrep filters, its pose is notably incorrect (12.1 $\AA$ RMSD from the crystallographic pose). The inhibitor coincidentally has similar substructures at both its ends: phthalazin-1(2H)-one and 3,4-dihydroisoquinolin-1(2H)-one, respectively. In the docked pose (Fig. 3c, green), the compound was flipped in the binding pocket relative to the crystallographic pose (Fig. 3c, pink), such that the phthalazin-1 $(2 H)$-one substructure satisfied the filters rather than the (correct) 3,4-dihydroisoquinolin$1(2 H)$-one substructure.
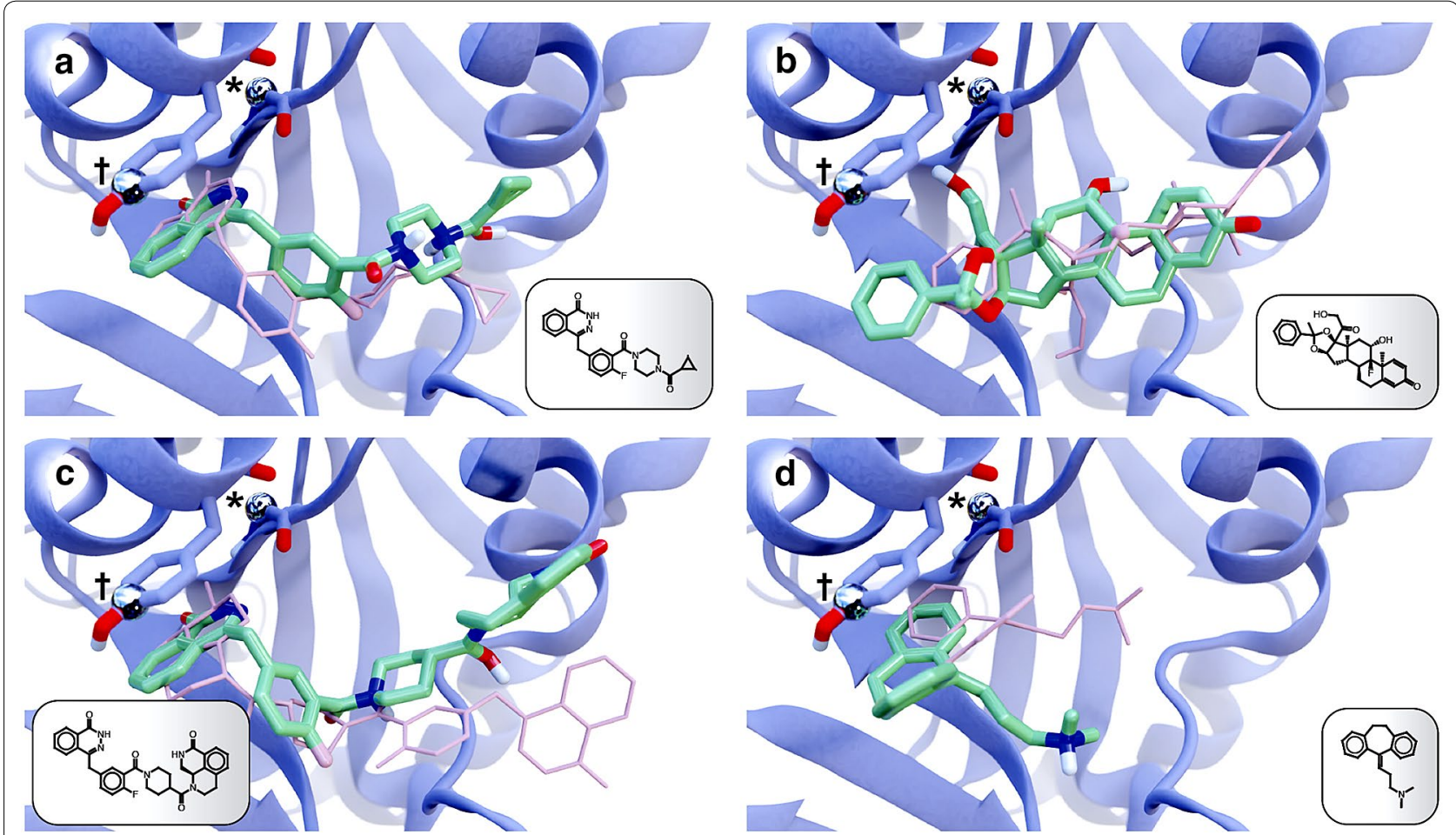

Fig. 3 Example poses taken from a benchmark HsPARP1 VS. Docked ligand poses are shown in green, and the HsPARP1 receptor (PDB: 6BHV) is shown in blue. The atoms used to define the hydrogen-bond and $\pi-\pi$ LigGrep filters (metallic spheres) are labeled with an asterisk and dagger, respectively. a Olaparib, with a generally correct docked pose that passed all LigGrep filters. The crystallographic pose is shown in pink. b Tricinolone acetophenonide (NSC37641), a high-scoring decoy molecule, had a top-scoring docked pose did not pass LigGrep filters (in pink), but a lower-scoring pose that did (in green). c Compound 33, with an incorrect docked pose that nevertheless passed LigGrep filters. The crystallographic pose is shown in pink. $\mathbf{d}$ Amitriptyline, a true ligand that could not have passed LigGrep filters, regardless of pose accuracy. The crystallographic pose is shown in pink 
Amitriptyline provides an example of the second disadvantage of the LigGrep approach. This low-micromolar HsPARP1 ligand ranked 229th out of 1561 compounds $(-9.2 \mathrm{kcal} / \mathrm{mol}$, 15th percentile) in our pre-LigGrep VS, and its docked and crystallographic poses were fairly similar (RMSD $3.8 \AA$ Å, Fig. 3d, green and pink, respectively) [76]. But amitriptyline lacks a nitrogen/oxygen atom adjacent to an aromatic ring and so could not ever pass our LigGrep filters, regardless of its pose.

\section{Comparison with existing programs}

Several powerful commercial docking programs also allow users to filter docked poses or to otherwise apply constraints during the docking process. LigGrep's main advantage is that it can be applied to VS performed with free, open-source docking programs that often lack builtin pose filters. In contrast, commercial programs are often expensive and have restrictive licenses that impose substantial commercialization and intellectual-property restrictions. Furthermore, in some cases license eligibility is regularly re-evaluated, making long-term access uncertain. We here compare two commercial programs to LigGrep and describe how LigGrep can complement their native functionality.

\section{Schrödinger Glide}

Schrödinger's Glide $[77,78]$ is a state-of-the-art commercial docking program that allows the user to apply constraints both during the docking process (such that they impact poses and scores) or after docking (as post-VS filters similar to LigGrep). Glide can account for (1) positional filters, which require a given docked-compound atom to occupy a user-defined spherical region; (2) excluded volumes, which require the compound to avoid defined regions of space; (3) nuclear Overhauser effect (NOE) constraints, which require a given protein/ligand atom-atom distance to fall within a user-provided range; and (4) hydrogen-bond/metal/metal-coordination constraints, which require the candidate ligand to form key interactions with receptor functional groups [79]. The positional and excluded-volume filters are notably similar to those that LigGrep implements.

We anticipate that most Glide users will prefer to use Glide's built-in constraints, but it is certainly possible to apply LigGrep filters to Glide-docked poses as well. Schrödinger's Maestro Suite can export protein receptor and small-molecule models as PDB and SDF files, respectively [78], formats that LigGrep can in turn accept as input.

\section{OpenEye FRED}

OpenEye's FRED is another popular commercial docking program that includes both protein and custom filters
[80, 81]. A FRED protein filter is satisfied when a docked compound is predicted to participate in a user-specified interaction (hydrogen bond, metal-chelator, contact, etc.) with a given protein atom. A custom filter is satisfied when a SMARTS-specified small-molecule substructure occupies a user-specified sphere. This last filter type in particular is very reminiscent of the LigGrep approach. As with Glide, FRED can also output docked poses in the SDF format, so LigGrep filters can be applied to FREDdocked poses as well.

\section{Conclusion}

LigGrep allows researchers performing VS to improve hit rates by leveraging prior knowledge about key receptor/ ligand interactions known to correlate with activity. Our results demonstrate that LigGrep can effectively filter out decoy molecules while retaining known ligands. In three separate test cases, LigGrep filtering improved hit rates over those obtained using AutoDock Vina alone. LigGrep will be a useful tool for the CADD community. We release it under the terms of the Apache License, Version 2.0. A copy is freely available at http://durrantlab.com/ liggrep/.

\section{Supplementary information}

Supplementary information accompanies this paper at https://doi. org/10.1186/s13321-020-00471-2.

Additional file 1: Table S1. Poly(ADP-ribose) polymerase 1 (PARP1) virtual screen, before and after applying two LigGrep filters (detailed results).

Table S2. H. sapiens peptidyl-prolyl cis-trans isomerase NIMA-interacting 1 (HsPin 1p) virtual screen, before and after applying a LigGrep filter (detailed results). Table S3. S. cerevisiae hexokinase-2 (ScHxk2p) virtual screen, before and after applying a LigGrep filter (detailed results). Table S4. AUROC and PAUROC values before and after LigGrep filtering. Figure S1. pROC curves describing our PARP1, HsPin $1 \mathrm{p}$, and $\mathrm{ScH}$ xk2 $\mathrm{p}$ VS, before (blue) and after (orange) applying LigGrep filters.

\section{Acknowledgements}

We thank Bhav Jain for helpful early-stage discussions and prototyping. We also acknowledge the University of Pittsburgh's Center for Research Computing for providing computer resources.

\section{Authors' contributions}

EJH and JDD wrote the final codebase, drafted the manuscript, and prepared the figures. CTL contributed to an early prototype of the codebase and critically reviewed/edited the manuscript. All authors read and approved the final manuscript.

\section{Funding}

This work was supported by the National Institute of General Medical Sciences of the National Institutes of Health [R01GM132353 to J.D.D.]. The content is solely the responsibility of the authors and does not necessarily represent the official views of the National Institutes of Health.

\section{Availability of data and materials}

Project name: LigGrep 1.0.0. Project home page: http://durrantlab.com/liggr ep. Operating systems: macOS, Linux, Windows. Programming language: Python 3. Other requirements: RDKit, NumPy, SciPy, Open Babel (optional). 
License: Apache License, Version 2.0. Restrictions to use by non-academics: None

\section{Competing interests}

The authors declare that they have no competing interests.

\section{Author details}

${ }^{1}$ Department of Biological Sciences, Carnegie Mellon University, Pittsburgh, PA 15213, United States. ${ }^{2}$ Department of Biological Sciences, University of Pittsburgh, Pittsburgh, PA 15260, United States.

Received: 10 July 2020 Accepted: 19 October 2020

Published online: 11 November 2020

\section{References}

1. Sliwoski G, Kothiwale S, Meiler J, Lowe EW (2014) Computational methods in drug discovery. Pharmacol Rev 66:334-95

2. Kontoyianni M (2017) Docking and virtual screening in drug discovery. Methods Mol Biol 1647:255-266

3. Lape M, Elam C, Paula S (2010) Comparison of current docking tools for the simulation of inhibitor binding by the transmembrane domain of the sarco/endoplasmic reticulum calcium ATPase. Biophys Chem 150:88-97

4. Ropp PJ, Spiegel JO, Walker JL, Green H, Morales GA, Milliken KA, Ringe JJ, Durrant JD (2019) Gypsum-DL: an open-source program for preparing small-molecule libraries for structure-based virtual screening. J Cheminform 11:34

5. Varghese JN, Smith PW, Sollis SL, Blick TJ, Sahasrabudhe A, McKimmBreschkin JL, Colman PM (1998) Drug design against a shifting target: a structural basis for resistance to inhibitors in a variant of influenza virus neuraminidase. Structure 6:735-46

6. Trott O, Olson AJ (2009) AutoDock Vina: improving the speed and accuracy of docking with a new scoring function, efficient optimization, and multithreading. J Comput Chem 31:455-461

7. Ropp P, Friedman A, Durrant JD (2017) Scoria: a Python module for manipulating 3D molecular data. J Cheminfor 9:52-58

8. O'Boyle NM, Banck M, James CA, Morley C, Vandermeersch T, Hutchison GR (2011) Open babel: an open chemical toolbox. J Cheminf 3:33

9. Landrum G, RDKit: open-source cheminformatics, Web Page

10. Oliphant TE (2006) Guide to NumPy. Brigham Young University, Provo

11. Jones E, Oliphant T, Peterson P et al (2001) SciPy: Open Source Scientific Tools for Python, Computer Program

12. Langelier M-F, Zandarashvili L, Aguiar PM, Black BE, Pascal JM (2018) NAD + analog reveals PARP-1 substrate-blocking mechanism and allosteric communication from catalytic center to DNA-binding domains. Nat Commun 9:844

13. Zhang $M$, Wang $X J$, Chen $X$, Bowman ME, Luo Y, Noel JP, Ellington AD, Etzkorn FA, Zhang Y (2012) Structural and kinetic analysis of prolylisomerization/phosphorylation cross-talk in the CTD code. ACS Chem Biol 7:1462-70

14. Kuser PR, Krauchenco S, Antunes OA, Polikarpov I (2000) The high resolution crystal structure of yeast hexokinase PII with the correct primary sequence provides new insights into its mechanism of action. J Bio Chem 275:20814-20821

15. Berman HM et al (2002) The protein data bank. Biol Crystallography Acta Crystallographica Sect D. https://doi.org/10.1107/S090744490 2003451

16. Berman HM, Westbrook J, Feng Z, Gilliland G, Bhat TN, Weissig H, Shindyalov IN, Bourne PE (2000) The protein data bank. Nucleic Acids Res 28:235-242

17. Gazgalis D, Zaka M, Abbasi BH, Logothetis DE, Mezei M, Cui M (2020) Protein binding pocket optimization for virtual high-throughput screening (vHTS) drug discovery. ACS Omega 5:14297-14307

18. Baumgartner MP, Camacho CJ (2016) Protein binding pocket optimization for virtual high-throughput screening (vHTS) drug discovery. J Chem Inform Modeling 56:1004-12

19. Ren J, Williams N, Clementi L, Krishnan S, Li WW (2010) Opal web services for biomedical applications. Nucleic Acids Res 38:W724-31
20. Dolinsky TJ, Nielsen JE, McCammon JA, Baker NA (2004) PDB2PQR: an automated pipeline for the setup of Poisson-Boltzmann electrostatics calculations. Nucleic Acids Res 32:W665-W667

21. Dolinsky TJ, Czodrowski P, Li H, Nielsen JE, Jensen JH, Klebe G, Baker NA (2007) PDB2PQR: expanding and upgrading automated preparation of biomolecular structures for molecular simulations. Nucleic Acids Res 35:W522-W525

22. Morris GM, Ruth H, Lindstrom W, Sanner MF, Belew RK, Goodsell DS, Olson AJ (2009) AutoDock4 and AutoDockTools4: automated docking with selective receptor flexibility. J Comput Chem. https://doi. org/10.1002/jcc.21256

23. National Cancer Institute. https://dtp.cancer.gov/organization/dscb/ obtaining/available_plates.htm. Accessed 30 Oct 2020

24. Liu T, Lin Y, Wen X, Jorissen RN, Gilson MK (2007) BindingDB: a webaccessible database of experimentally determined protein-ligand binding affinities. Nucleic Acids Res 35:D198-201

25. Chen X, Lin Y, Liu M, Gilson MK (2002) The binding database: data management and interface design. Bioinformatics 18:130-139

26. Kochnev Y, Hellemann E, Cassidy KC, Durrant JD (2020) Webina: an open-source library and web app that runs AutoDock vina entirely in the web browser. Bioinformatics. https://doi.org/10.1093/bioinforma tics/btaa579

27. Spiegel JO, Durrant JD (2020) AutoGrow4: an open-source genetic algorithm for de novo drug design and lead optimization. J Cheminform 12:1-16

28. Thorsell A-G, Ekblad T, Karlberg T, Löw M, Pinto AF, Trésaugues L, Moche M, Cohen MS, Schuler H (2017) Structural Basis for potency and promiscuity in poly(ADP-ribose) polymerase (PARP) and tankyrase inhibitors. J Med Chem 60:1262-1271

29. Kinoshita T, Nakanishi I, Warizaya M, Iwashita A, Kido Y, Hattori K, Fujii T (2004) Inhibitor-induced structural change of the active site of human poly(ADP-ribose) polymerase. FEBS Lett 556:43-6

30. Zhang Y, Daum S, Wildemann D, Zhou XZ, Verdecia MA, Bowman ME, Lucke C, Hunter T, Lu K-P, Fischer G, Noel JP (2007) Structural basis for high-affinity peptide inhibition of human Pin1. ACS Chem Biol 2:320-8

31. Pettersen EF, Goddard TD, Huang CC, Couch GS, Greenblatt DM, Meng EC, Ferrin TE (2004) UCSF chimera-a visualization system for exploratory research and analysis. J Comput Chem 25:1605-1612

32. Chaudhuri AR, Nussenzweig A (2017) The multifaceted roles of PARP1 in DNA repair and chromatin remodelling. Natu Rev Mol Cell Biol 18:610

33. Wang L, Liang C, Li F, Guan D, Wu X, Fu X, Lu A, Zhang G (2017) PARP1 in carcinomas and PARP1 inhibitors as antineoplastic drugs. Int J Mol Sci. https ://doi.org/10.3390/ijms18102111

34. Jiang X, Li W, Li X, Bai H, Zhang Z (2019) Current status and future prospects of PARP inhibitor clinical trials in ovarian cancer. Cancer Manag Res 11:4371-4390

35. Malyuchenko NV, Kotova EY, Kulaeva OI, Kirpichnikov MP, Studitskiy VM (2015) PARP1 inhibitors: antitumor drug design. Acta Naturae 7:27-37

36. Montoni A, Robu M, Pouliot E, Shah GM (2013) Resistance to PARP-inhibitors in cancer therapy. Front Pharmacol 4:18

37. Ye N, Chen C-H, Chen T, Song Z, He J-X, Huan X-J, Song S-S, Liu Q, Chen Y, Ding J, Xu Y, Miao Z-H, Zhang A (2013) Design, synthesis, and biological evaluation of a series of benzo[de][1,7]naphthyridin-7(8H)-ones bearing a functionalized longer chain appendage as novel PARP1 inhibitors. J Med Chem 56:2885-903

38. Chen Y, Wu Y-R, Yang H-Y, Li X-Z, Jie M-M, Hu C-J, Wu Y-Y, Yang S-M, Yang Y-B (2018) Prolyl isomerase Pin1: a promoter of cancer and a target for therapy. Cell Death Dis 9:883

39. Göthel SF, Marahiel MA (1999) Peptidyl-prolyl cis-trans isomerases, a superfamily of ubiquitous folding catalysts. Cell Mol Life Sci 55:423-36

40. Takahashi K, Uchida C, Shin R-W, Shimazaki K, Uchida T (2008) Prolyl isomerase, Pin 1: new findings of post-translational modifications and physiological substrates in cancer, asthma and Alzheimer's disease. Cell Mol Life Sci 65:359-75

41. Lu KP, Finn G, Lee TH, Nicholson LK (2007) Prolyl cis-trans isomerization as a molecular timer. Nat Chem Biol 3:619-29

42. D’Artista L, Bisso A, Piontini A, Doni M, Verrecchia A, Kress TR, Morelli MJ, Del Sal G, Amati B, Campaner S (2016) Pin1 is required for sustained B cell proliferation upon oncogenic activation of Myc. Oncotarget 7:21786-98 
43. Wulf G, Garg P, Liou Y-C, Iglehart D, Lu KP (2004) Modeling breast cancer in vivo and ex vivo reveals an essential role of Pin1 in tumorigenesis. EMBO J 23:3397-407

44. Girardini JE et al (2011) A Pin1/mutant p53 axis promotes aggressiveness in breast cancer. Cancer Cell 20:79-91

45. Guo C, Hou X, Dong L, Marakovits J, Greasley S, Dagostino E, Ferre R, Johnson MC, Humphries PS, Li H, Paderes GD, Piraino J, Kraynov E, Murray BW (2014) Structure-based design of novel human Pin1 inhibitors (III): optimizing affinity beyond the phosphate recognition pocket. Bioorg Med Chem Lett 24:4187-91

46. Pollard-Knight D, Cornish-Bowden A (1982) Mechanism of liver glucokinase. Mol Cell Biochem 44:71-80

47. Lunt SY, Vander Heiden MG (2011) Aerobic glycolysis: meeting the metabolic requirements of cell proliferation. Ann Rev Cell Dev Biol 27:441-464

48. Pastorino JG, Hoek JB (2003) Hexokinase II: the integration of energy metabolism and control of apoptosis. Curr Med Chem 10:1535-1551

49. Mathupala SP, Ko YH, Pedersen PL (2009) Seminars in cancer biology. Semin Cancer Biol 19:17-24

50. Feron O (2009) Pyruvate into lactate and back: from the Warburg effect to symbiotic energy fuel exchange in cancer cells. Radiothe Oncol 92:329-333

51. Pavlova NN, Thompson CB (2016) The emerging hallmarks of cancer metabolism. Cell Metab 23:27-47

52. Phan LM, Yeung S-CJ, Lee M-H (2014) Cancer metabolic reprogramming: importance, main features, and potentials for precise targeted anti-cancer therapies. Cancer Biology Med 11:1

53. Vander Heiden MG (2011) Targeting cancer metabolism: a therapeutic window opens. Nat Rev Drug Discov 10:671-684

54. Teicher BA, Linehan WM, Helman $\sqcup$ (2012) Targeting cancer metabolism. Clin Cancer Res 18:5537-5545

55. Denko NC (2008) Hypoxia, HIF1 and glucose metabolism in the solid tumour. Nat Rev Cancer 8:705-713

56. Liberti MV, Locasale JW (2016) The Warburg effect: how does it benefit cancer cells? Trends Biochem Sci 41:211-218

57. Mathupala SP, Rempel A, Pedersen PL (1997) Aberrant glycolytic metabolism of cancer cells: a remarkable coordination of genetic, transcriptional, post-translational, and mutational events that lead to a critical role for type II hexokinase. J Bioenergetics Biomembranes 29:339-343

58. Pelicano H, Martin D, Xu R, Huang P (2006) Glycolysis inhibition for anticancer treatment. Oncogene 25:4633-4646

59. Bao F, Yang K, Wu C, Gao S, Wang P, Chen L, Li H (2018) New natural inhibitors of hexokinase 2 (HK2): steroids from Ganoderma sinense. Fitoterapia 125:123-129

60. Granchi C, Fancelli D, Minutolo F (2014) An update on therapeutic opportunities offered by cancer glycolytic metabolism. Bioorganic Med Chem Lett 24:4915-4925

61. Lin H, Zeng J, Xie R, Schulz MJ, Tedesco R, Qu J, Erhard KF, Mack JF, Raha K, Rendina AR et al (2016) Discovery of a novel 2,6-disubstituted glucosamine series of potent and selective hexokinase 2 inhibitors. ACS Med Chem Lett 7:217-222

62. Hampton A, Hai TT, Kappler F, Chawla RR (1982) Species-and isozymespecific enzyme inhibitors. 6. synthesis and evaluation of two-substrate condensation products as inhibitors of hexokinases and thymidine kinases. J Med Chem 25:801-805

63. Hampton A, Picker D, Nealy KA, Maeda M (1982) Use of adenine nucleotide derivatives to assess the potential of exo-active-site-directed reagents as species-or isozyme-specific enzyme inactivators. 4. Interactions of adenosine 5'-triphosphate derivatives with adenylate kinases from Escherichia coli and rat tissues. J Med Chem. 25:382-386
64. Yamaguchi R, Janssen E, Perkins G, Ellisman M, Kitada S, Reed JC (2011) Efficient elimination of cancer cells by deoxyglucose-ABT-263/737 combination therapy. PloS One 6:e24102

65. Zhang XD, Deslandes E, Villedieu M, Poulain L, Duval M, Gauduchon P, Schwartz L, Icard P (2006) Effect of 2-deoxy-D-glucose on various malignant cell lines in vitro. Anticancer Res 26:3561-3566

66. Aft RL, Lewis JS, Zhang F, Kim J, Welch MJ (2003) Enhancing targeted radiotherapy by copper (II) diacetyl-bis (N4-methylthiosemicarbazone) using 2-deoxy-D-glucose. Cancer Res 63:5496-5504

67. Maher JC (2006) Treatment of tumor cells with the glycolytic inhibitor, 2-deoxy-D-glucose: effects and mechanisms of resistance. Ph.D. Thesis, University of Miami

68. Zhang D, Li J, Wang F, Hu J, Wang S, Sun Y (2014) 2-Deoxy-D-glucose targeting of glucose metabolism in cancer cells as a potential therapy. Cancer Lett 355:176-183

69. Morrow J, De Carli L (1967) The correlation of resistance to 2-deoxyglucose with alkaline phosphatase levels in a human cell line. Exp Cell Res 47:1-11

70. Defenouillère Q, Verraes A, Laussel C, Friedrich A, Schacherer J, Léon S (2019) The induction of HAD-like phosphatases by multiple signaling pathways confers resistance to the metabolic inhibitor 2-deoxyglucose. Science Signal 12:eaaw8000

71. Stein M, Lin H, Jeyamohan C, Dvorzhinski D, Gounder M, Bray K, Eddy S, Goodin S, White E, DiPaola RS (2010) Targeting tumor metabolism with 2-deoxyglucose in patients with castrate-resistant prostate cancer and advanced malignancies. Prostate 70:1388-1394

72. Zhang J et al (2017) c-Src phosphorylation and activation of hexokinase promotes tumorigenesis and metastasis. Nat Commun 8:13732

73. Anderson CM, Stenkamp RE, Steitz TA (1978) Sequencing a protein by X-ray crystallography: II. Refinement of yeast hexokinase B Co-ordinates and sequence at $2.1 \AA$ resolution. J Mol Biol 123:15-33

74. Kuser P, Cupri F, Bleicher L, Polikarpov I (2008) Crystal structure of yeast hexokinase PI in complex with glucose: A classical "induced fit" example revised, Proteins: Structure. Function Bioinform 72:731-740

75. Dawicki-McKenna JM, Langelier M-F, DeNizio JE, Riccio AA, Cao CD, Karch KR, McCauley M, Steffen JD, Black BE, Pascal JM (2015) PARP-1 activation requires local unfolding of an autoinhibitory domain. Mol Cell 60:755-768

76. Fu L, Wang S, Wang X, Wang P, Zheng Y, Yao D, Guo M, Zhang L, Ouyang $L$ (2016) Crystal structure-based discovery of a novel synthesized PARP1 inhibitor (OL-1) with apoptosis-inducing mechanisms in triple-negative breast cancer. Sci Rep 6:3

77. Repasky MP, Shelley M, Friesner RA (2007) Flexible ligand docking with Glide. Curr Protocols Bioinform 18:8-12

78. Schrödinger. https://www.schrodinger.com/documentation. Accessed 30 Oct 2020

79. Schrödinger. https://www.schrodinger.com/kb/186. Accessed 20 June 2020

80. OpenEye Scientific Software. https://docs.eyesopen.com/applications/ oedocking/. Accessed 30 Oct 2020

81. OpenEye Scientific Software. https://docs.eyesopen.com/applications/ oedocking/make_receptor/make_receptor_setup.html. Accessed 22 June 2020

\section{Publisher's Note}

Springer Nature remains neutral with regard to jurisdictional claims in published maps and institutional affiliations. 